\title{
Mirror Quality and the Performance of Reflective Arrayed-Waveguide Grating Multiplexers
}

\author{
A. A. Bernussi, L. Grave de Peralta, V. Gorbounov, J. A. Linn, S. Frisbie, R. Gale, and H. Temkin, Fellow, IEEE
}

\begin{abstract}
This paper discusses an investigation of the effects of mirror quality on the performance of reflecting arrayed-waveguide-grating multiplexers. Using specially designed external mirrors as external reflectors, the optimum flatness and microroughness of the reflecting surface are determined. The experiments discussed in the paper indicate that a reflecting-surface flatness of $\lambda / 4$ is sufficient to obtain high-performance reflective multiplexers. Extrinsic mirror-related phase and intensity errors were found to be negligible for surfaces polished with diamond lapping films with a grain size smaller than $1 \mu \mathrm{m}$. These experimental results were found to be in excellent agreement with simulations based on mirror-related changes in the waveguide-grating-length increment.
\end{abstract}

Index Terms-Arrayed-waveguide grating (AWG), multiplexer, silica waveguides, wavelength-division multiplexing (WDM).

\section{INTRODUCTION}

D IFFERENT approaches to the design of arrayed-waveguide grating (AWG) multiplexers, combined with different choices of materials, have been reported in the past few years [1]-[6]. Reflective AWGs (R-AWGs) have been proposed and demonstrated as one of the approaches resulting in more compact devices [7]-[12]. However, the fabrication of R-AWGs includes preparation of a reflecting surface used to fold the grating. Thus, in addition to inaccuracies in the device fabrication process, common to AWG devices, the performance of R-AWGs can be affected by the nonflatness and microroughness of the reflecting surface terminating the grating.

This paper presents detailed experimental investigation of the effects of the optical quality of the reflecting surface on the transmission response of R-AWGs. The smoothness required of the reflecting surface was determined using an external mirror polished to different levels. A specially designed external mirror, the curvature of which can be precisely and controllably varied, was used to establish the flatness necessary to obtain high-performance R-AWGs. It is shown that the reflecting surface with a flatness of $\lambda / 4$ and polished with diamond lapping films with grain sizes smaller than $1 \mu \mathrm{m}$ is sufficient to avoid introduction of any measurable degradation in the performance of the device. Any limitations in the device performance are ultimately determined by intrinsic contributions to phase and intensity errors, as is the case with conventional AWGs.

Manuscript received September 25, 2003; revised December 2, 2003. This work was supported by the State of Texas under the Technology Development and Transfer program and the Jack F. Maddox Foundation.

The authors are with the Department of Electrical and Computer Engineering, Texas Tech University, Lubbock, TX 79409 USA.

Digital Object Identifier 10.1109/JLT.2004.831196

\section{Design, FAbrication, AND Performance of R-AWGs}

We have modified the conventional AWG by folding it with a reflecting surface [10]-[12]. The Gaussian transmission response R-AWG is designed for multiplexing 40 channels with a separation of $100 \mathrm{GHz}$. The device consists of the input-output (I/O) coupler, the free-space propagation region, the waveguide grating, and the reflecting surface. The I/O coupler contains one input waveguide and 40 output waveguides. The main advantage of this modification is that the resulting grating of the R-AWG is very compact and the device can be fabricated in a conventional silicon integrated-circuit (IC) foundry. Front-end photolithography in such foundries is done with projection steppers. These are designed to expose a fixed area, typically less than $2 \times 2 \mathrm{~cm}^{2}$, at a time. The grating of a conventional (nonreflective) AWG is simply too large to be printed with a stepper. Stitching between fields cannot be done precisely without considerable effort and expensive changes in standard IC foundry procedures. In our design, a grating with 330 waveguides readily fits into the area of $1.5 \times 1.8 \mathrm{~cm}^{2}$ and can thus be easily printed with conventional photolithographic steppers.

The R-AWGs discussed in this paper are based on P- and B-doped silica waveguides with the relative refractive index $(n)$ difference $\left(n_{\text {core }}=1.456\right.$ and $n_{\text {cladding }}=1.446$ at $\lambda=$ $1.55 \mu \mathrm{m})$ between the core and the cladding layers of $0.68 \%$ [10]. The waveguide cores are $5 \mu \mathrm{m}$ thick and are etched to produce $6-\mu \mathrm{m}$-wide ridges. The constant path length difference $(\Delta L)$ between grating waveguides $\Delta L=12.3 \mu \mathrm{m}$ is half that of similar nonreflective device. This corresponds to a diffraction order $m=23$. This small $\Delta L$ value enables us to place as many as 330 waveguides in the grating, while maintaining $30-\mu \mathrm{m}$ separation between adjacent waveguides at the reflecting surface.

Devices were measured after chip separation, diamond polishing, and deposition of a reflecting metal film on the mirror surface. High reflectivity of the mirror was assured by the deposition of a $\mathrm{Cr}-\mathrm{Au}$ film. The average insertion loss of $<-2.5$ $\mathrm{dB}$, a loss nonuniformity less than $1.0 \mathrm{~dB}$, crosstalk of $30 \mathrm{~dB}$, a polarization-dependent wavelength shift of less than $0.02 \mathrm{~nm}$, and a polarization-dependent loss of $\sim 0.1 \mathrm{~dB}$, were obtained. In the experiments discussed in this paper, the $\mathrm{Cr}-\mathrm{Au}$ film was removed from the reflecting surface, and an external mirror was used instead as a reflector. In our R-AWG design, the waveguides of the grating are perpendicular to the reflecting surface. In all experiments described in this paper, the angular deviation of the external mirror relative to the reflecting surface integral to the device was $\ll 1^{\circ}$. This results in negligible polarization-dependent penalties at the mirror surface. 


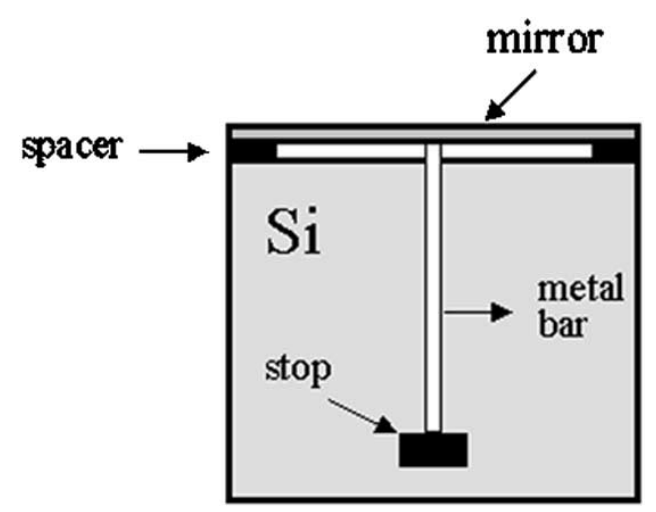

Fig. 1. Schematic illustration of the mirror assembly used to evaluate deviations in the reflecting surface flatness. The metal rod is $\sim 1.5 \mathrm{~mm}$ diameter and $10 \mathrm{~mm}$ long.

\section{EXTERNAL REFLECTOR-EXPERIMENTAL RESULTS AND DISCUSSION}

The optical quality of the reflecting surface is of fundamental importance in R-AWGs. Deviations in flatness and the presence of microroughness and defects, such as scratches and digs created during the polishing step, can be a source of increased losses, larger transmission channel spectral bandwidth, and increased crosstalk between output channels. Using external mirrors as reflectors, instead of the reflecting surface integral to the R-AWG, it is possible to determine the flatness and the optical quality requirements necessary to obtain high-performance device operation.

\section{A. Flatness of the Reflecting Surface}

In order to evaluate the effects of the reflecting surface flatness on the performance of R-AWGs, we have constructed an external mirror the curvature of which can be precisely controlled. A schematic drawing of the mirror assembly is shown in Fig. 1. It consists of a rectangular section of an $\mathrm{Si}$ wafer, used as a base, and a polished stripe of $\mathrm{Si}$, used as a mirror. The mirror is attached to the edges of the base by two spacers, also made of Si. A metal bar connects the base and the mirror stripe. The bar is attached with epoxy to the mirror and to a small piece of $\mathrm{Si}$, a stop, mounted on the base wafer. The shape of the reflecting surface is modified by changing the temperature of the mirror assembly. When the temperature is increased (decreased), the metal rod elongates (shortens) bending the mirror surface. The temperature-induced deformation of the external mirror was first calibrated by interferometric microscopy (using a WYKO NT-1100 optical profiler). In these experiments, the mirror assembly was placed on a temperature-controlled stage. Examples of bending profiles at three different temperatures are shown in Fig. 2. This figure clearly shows that the mirror changes from concave, at temperatures lower than $35^{\circ} \mathrm{C}$, to convex, at higher temperatures. Effective radius of a curvature $>100 \mathrm{~m}$ was determined for the temperature of $35^{\circ} \mathrm{C}$. This corresponds to the flatness of $\lambda / 4$.

The performance of R-AWGs with the external mirror illustrated in Fig. 1 was investigated as a function of temperature. Both the R-AWG and the external mirror assembly were mounted on a $\mathrm{Si}$ submount placed over a temperature-con-

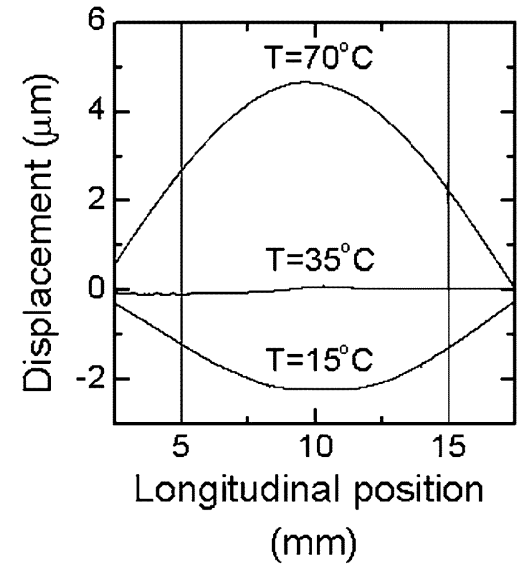

Fig. 2. Examples of temperature-induced bending profiles measured for the assembly mirror shown in Fig. 1. Vertical lines were drawn to indicate the grating waveguides reflecting surface region.

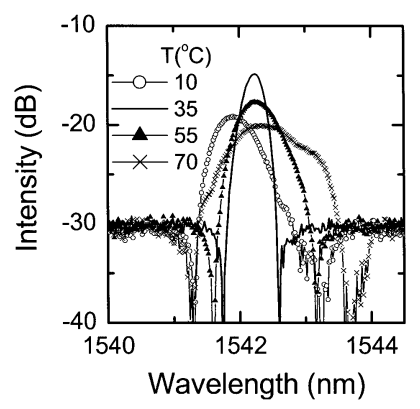

Fig. 3. Single-channel transmission spectra of an R-AWG at different temperatures using the mirror assembly illustrated in Fig. 1 as the reflector. The reflecting surface of the external mirror was not metallized, resulting in higher losses.

trolled stage. The external mirror was positioned closely (within $10 \mu \mathrm{m})$ to the surface terminating the grating of the R-AWG. Single-channel transmission response spectra, at different temperatures, are plotted in Fig. 3. The output spectra vary significantly with changes in the temperature-induced curvature of the external mirror. Similar results were obtained for other output channels of the R-AWG. At temperatures below or above $\mathrm{T}=35^{\circ} \mathrm{C}$, for which the mirror is not flat, additional losses and broadening of the transmission spectra were observed. The shift of the center peak wavelength observed at different temperatures in Fig. 3 is attributed to the temperature dependence of the refractive index of the silica glass forming the device [1]. The broadening and asymmetry in the peak response observed for $\mathrm{T} \neq 35^{\circ} \mathrm{C}$ are due to pronounced phase errors caused by the mirror curvature [13], [14]. The corresponding center channel peak loss and 3-dB bandwidth changes (relative to the measurement performed at $\mathrm{T}=35^{\circ} \mathrm{C}$ ) are plotted as a function of temperature in Fig. 4(a) and (b), respectively. Variation in the mirror curvature results in changes in the insertion loss as large as $\sim 4.5 \mathrm{~dB}$, as shown in Fig. 4(a). The 3-dB bandwidth is also affected by the mirror curvature. In the temperature range of $35-70{ }^{\circ} \mathrm{C}$, the $3-\mathrm{dB}$ bandwidth increased by $0.74 \mathrm{~nm}$, as plotted in Fig. 4(b). Minimum insertion losses and 3-dB bandwidth were obtained at $\mathrm{T}=35^{\circ} \mathrm{C}$. This is consistent with the results of Fig. 2, where we determined the optimum surface flatness of the mirror assembly. 


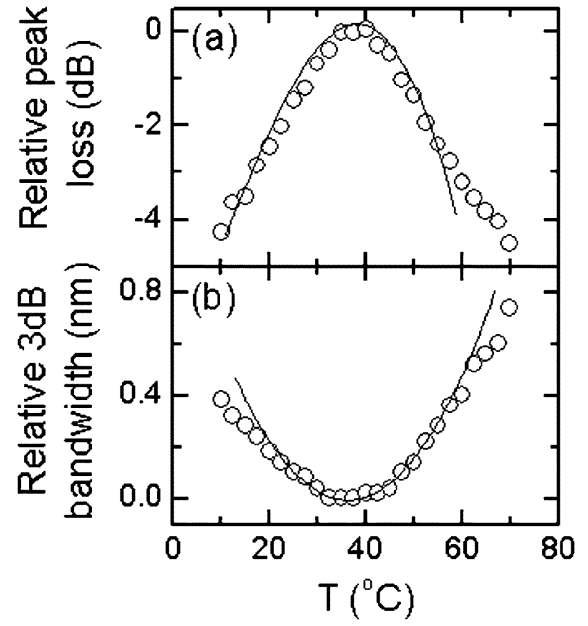

Fig. 4. Calculated (-) and experimental (o) relative peak loss (a) and relative $3-\mathrm{dB}$ channel bandwidth (b) as a function of temperature, using as a reflector the mirror assembly illustrated in Fig. 1.

The degradation in the transmission performance of the R-AWG with increasing curvature of the external mirror (Figs. 3 and 4) arises from nonconstant contributions to the consecutive waveguide path-length difference $\Delta L$ for which the device was designed. When the external mirror is curved, the distance between the reflecting surface terminating the grating and the external mirror is no longer constant. As a result, the additional path-length difference introduced at the air gap is no longer constant. A variation in the path-length difference between consecutive waveguides of the grating results in additional phase errors [14], degrading the transmission response of the R-AWG. In contrast, when the surface of the external mirror is flat, the path-length difference remains constant, resulting in no performance degradation, as shown in Fig. 4.

In order to analyze the effect of the external mirror curvature on the R-AWG output characteristics, we simulated the device response using the AWG field model in the Gaussian approximation [12], [15]. Using the AWG design parameters and assuming that the temperature-induced mirror deformation results in a parabolic change in $\Delta L$, we can calculate singlechannel transmission spectra. A comparison between simulated and measured relative peak losses and 3-dB bandwidth, as a function of the temperature, is shown in Fig. 4. A good agreement between theory and experiment is clear. The discrepancy between calculated and measured AWG parameters observed for $\mathrm{T}>60{ }^{\circ} \mathrm{C}$ is attributed to nonhomogeneous deformation of the mirror assembly at high temperatures.

Measurements on several R-AWGs (not shown) with the $\mathrm{Cr}-\mathrm{Au}$ film directly deposited on the grating-terminating surface were performed in order to evaluate the effects of the flatness of this surface after the diamond-polishing step. Our results show the same $3-\mathrm{dB}$ bandwidth as the minimum obtained in Fig. 4(b). These experiments also show very low insertion losses of less than $-2.5 \mathrm{~dB}$. This is consistent with the surface better than $\lambda / 4$ measured in a separate experiment, also by phase contrast microscopy, on reflecting surface formed directly on R-AWGs. Our standard diamond-polishing step used in device fabrication is thus sufficient to ensure high-performance of R-AWGs.

\section{B. Optical Quality of the Mirror Surface}

Grating waveguides of the R-AWGs investigated in this paper were designed to be perpendicular to the reflecting surface. In addition, each waveguide in the grating terminates in a straight segment at the mirror surface. This was done in order to preserve a constant path-length difference between consecutive waveguides even after dicing and polishing. However, in practice, deviations from the design path length may occur as a result of intrinsic or extrinsic fabrication errors. Intrinsic error sources involve device processing, material inhomogeneities, and finite photomask resolution [12], [14]. In the case of R-AWGs, extrinsic errors are associated with imperfections in the flatness and polish of the reflecting surface and any inhomogeneities in the high-reflectivity material deposited at this surface. As shown previously, deviations in the path-length difference between consecutive waveguides in the grating contribute to degradation of the insertion loss, the 3-dB bandwidth, and the crosstalk of an AWG [13], [14].

In the fabrication of R-AWGs studied here, mechanical polishing was used to obtain optical grade reflectors terminating the grating. The reflecting surface was polished sequentially with lapping films with decreasing diamond grain sizes $\left(d_{s}\right)$, from 15.0 to $0.1 \mu \mathrm{m}$. In order to study the effects of the polish on the performance of R-AWGs, we experimented with an external mirror placed within $5 \mu \mathrm{m}$ to the reflecting surface integral to the device. The external mirror was formed at the edge of a silicon wafer, polished with diamond lapping films. In these experiments, the external mirror was not coated with high-reflectivity material, and index-matching fluid was applied between the mirror and the device.

The quality of the polished surfaces was evaluated using optical profiler microscopy and atomic force microscopy (AFM). An example of a top-view optical profiler image obtained on a surface of a mirror polished with $d_{s}=3.0 \mu \mathrm{m}$ is shown in Fig. 5(a). The surface shows a pattern of parallel peaks and valleys along the entire mirror area. The corresponding vertical and horizontal cross-sectional profiles, obtained at $x=20 \mu \mathrm{m}$ and $y=20 \mu \mathrm{m}$, are shown in Fig. 5(b) and (c), respectively. The maximum surface roughness $\left(R_{\max }\right)$ of $\sim 60 \mathrm{~nm}$ was determined from both cross sections. When the surface was finished with $d_{s} \leq 0.5 \mu \mathrm{m}, R_{\max }<15 \mathrm{~nm}$ was determined. When the mirror surface is polished with larger $\mathrm{d}_{\mathrm{s}}$ values, for example $\mathrm{d}_{\mathrm{s}}=9.0 \mu \mathrm{m}, R_{\max }$ as large as $2.0 \mu \mathrm{m}$ could be seen. Surface roughness of this magnitude can contribute to large phase and intensity errors, degrading the performance of the device, as discussed hereafter.

Fig. 6 shows examples of the transmission spectra of a single output channel of an R-AWG operated with an external mirror. The mirror surface was finished using diamond lapping films with $d_{s}$ of $0.1,1.0$, and $9.0 \mu \mathrm{m}$. The spectra corresponding to mirrors polished with $d_{s}=0.1$ and $1.0 \mu \mathrm{m}$ are very similar. The insertion loss increased when the external mirror was polished with $d_{s}=9.0 \mu \mathrm{m}$. In addition, the relative intensity of subsidiary sidelobes became more pronounced for the surface 

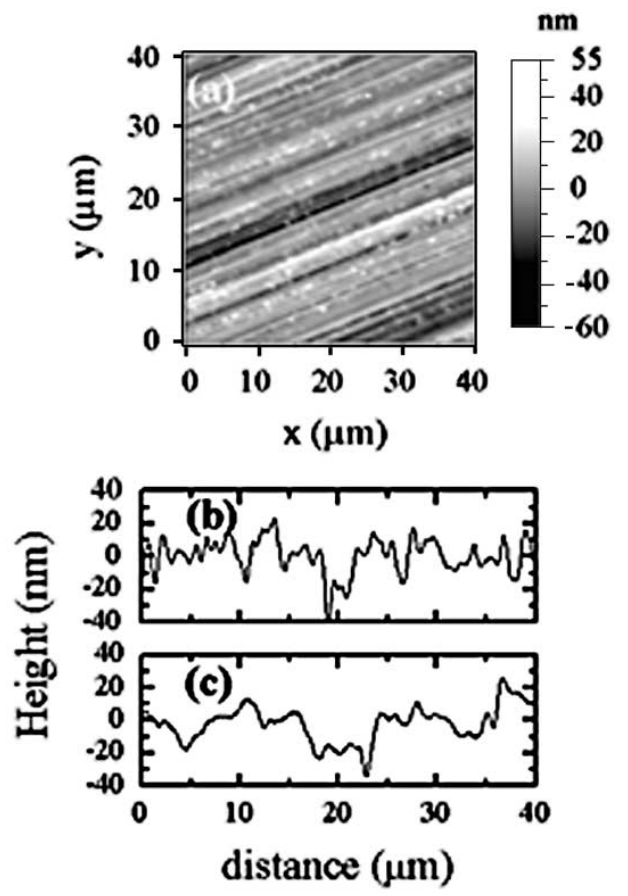

Fig. 5. (a) Optical image profiler of a mirror surface polished with $d_{s}=3.0 \mu \mathrm{m}$. Vertical (b) and horizontal (c) roughness cross-sectional profiles obtained at $x=20 \mu \mathrm{m}$ and $y=20 \mu \mathrm{m}$, respectively.

polished with $d_{s}=9.0 \mu \mathrm{m}$. The 3-dB bandwidth is identical for transmission spectra of R-AWGs measured with external mirrors polished with $d_{s}=0.1$ and $1.0 \mu \mathrm{m}$. A slight increase $(\sim 10 \%)$ in the 3-dB bandwidth was observed when the external mirror was polished with $d_{s}=9.0 \mu \mathrm{m}$. Similar results were found for other output channels of the device. The differences in the performance of the R-AWG shown in Fig. 6 are attributed to increasing microroughness of polished surfaces. Mirrors polished with $d_{s} \leq 3 \mu \mathrm{m}$ exhibited mirror-like surfaces with a small density of defects. In contrast, surfaces polished with $d_{s}>3 \mu \mathrm{m}$ resulted in rougher surfaces (see Fig. 5) and lower reflectivity. The roughness of the mirror polished with $d_{s}=9.0 \mu \mathrm{m}$ contributes to larger changes in the path-length difference between consecutive waveguides in the grating as well as to light scattering at the reflective surface. This results in additional losses at the reflecting surface and increased crosstalk (see Fig. 6).

In order to further understand the effect of the mirror quality on the response of the R-AWG, we calculate the output spectra, taking into account additional contributions to $\Delta L$ due to the microroughness of the external mirror. We consider microroughness to be the source of extrinsic errors. The fluctuations in $\Delta L$ are modeled assuming contributions from the intrinsic errors, due to the photomask resolution $(\delta)$ and the extrinsic errors $(\sigma)$. Changes in $\Delta L$ are calculated using a uniform pseudorandom distribution with standard deviation given by $(\delta+\sigma)$. In the case of a perfectly smooth surface, the deviation in $\Delta L$ is limited to $\delta=0.1 \mu \mathrm{m}(\sigma=0)$, corresponding to the resolution of the photomask used in the fabrication of our R-AWGs [12]. The upper limit on $\sigma$ is determined by the microroughness of the polished surface.

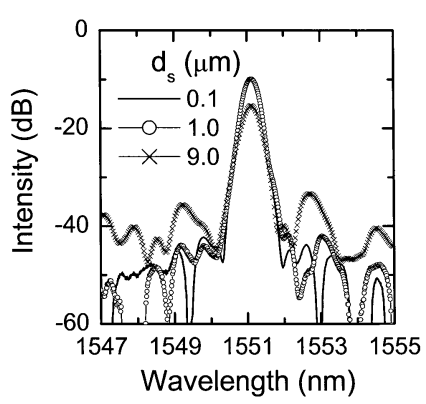

Fig. 6. Transmission spectra of a single output channel of an R-AWG using external mirrors polished with different $d_{s}$ values. The reflecting surface of the external mirror was not metallized, resulting in higher losses.

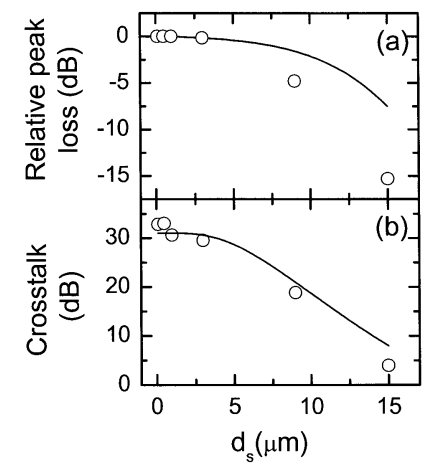

Fig. 7. Calculated (一) and experimental (o) crosstalk (a) and relative peak loss (b) as a function of $d_{s}$.

A correlation between the surface microroughness and the width of the waveguide $\left(w_{\mathrm{wg}}\right)$ must be considered. If $d_{s}<w_{\mathrm{wg}}$, the number of polishing-induced peaks and valleys $(N)$ within each waveguide of the grating is given by $N_{\sim} w_{\mathrm{wg}} / d_{s}$. We assume here a uniform pseudorandom distribution of peaks and valleys with amplitudes in the range $\pm R_{\max } / 2$, consistent with the data of Fig. 5. For $d_{s}>w_{\mathrm{wg}}$ only a single peak or valley contributes to the change in $\Delta L$. As a result, $\Delta L$ is modified by a random increment determined by the average of the amplitude of the $N$ peaks and valleys at the polished edge. The standard deviation $\sigma$ of the distribution of modified $\Delta L \mathrm{~s}$ can be then calculated for each $d_{s}$. For a waveguide with $w_{\mathrm{wg}}=6 \mu \mathrm{m}$ and surfaces polished with $d_{s}=3.0$ and $9.0 \mu \mathrm{m}$, we determine $\sigma=10 \mathrm{~nm}$ and $0.5 \mu \mathrm{m}$, respectively.

Fig. 7 compares the measured and calculated changes in the peak loss and crosstalk as a function of $d_{s}$. The insertion loss is practically constant $(<0.5 \mathrm{~dB})$ for external mirrors polished with $d_{s} \leq 3.0 \mu \mathrm{m}$. Increased loss is seen for $d_{s}>3.0 \mu \mathrm{m}$ as a result of larger phase errors and greater microroughness. This results in higher light scattering at the reflecting surface and increased losses. The discrepancy between the calculated and measured peak loss change [Fig. 7(a)] for larger values of $d_{s}$ is attributed to intensity errors (mainly for $d_{s}>3.0 \mu \mathrm{m}$ ). Since the exact description of light scattering at polished surfaces with various degrees of microroughness is not straightforward, we did not include intensity errors in our simulation. The crosstalk [Fig. 7(b)] is also influenced by phase and intensity errors introduced during mirror polishing [14]. Good agreement between calculated and experimental values of crosstalk is 
clearly observed over the entire range of $d_{s}$ values. This is attributed to lower influence of intensity errors on the crosstalk performance. Similar values of crosstalk were obtained for mirrors polished with $d_{s}=0.1$ and $0.5 \mu \mathrm{m}$. We conclude that the polishing step carried out with diamond lapping with films $d_{s} \sim 0.1-0.5 \mu \mathrm{m}$ does not contribute to significant extrinsic phase and intensity errors. Larger values of $d_{s}(>3.0 \mu \mathrm{m})$ resulted in increased crosstalk.

\section{CONCLUSION}

Effects of the flatness and roughness of the reflecting surface terminating the grating of the R-AWG on its performance were investigated. We show that the polishing step used in the fabrication of R-AWGs, carried out with diamond lapping films with $d_{s}<1 \mu \mathrm{m}$, does not introduce significant extrinsic phase or intensity errors. This is supported by measurements of the transmission response of R-AWGs using external mirrors polished with diamond lapping films with different grain sizes. The results indicate that the performance of R-AWGs is limited fundamentally by intrinsic errors accrued during the device fabrication. The performance of both the conventional AWGs and the R-AWG discussed in this paper is thus limited by the same mechanism.

An external reflector, with a surface flatness that could be controlled very precisely, was constructed and used in the present study. From these experiments, it was determined that a mirror flatness of $\lambda / 4$ is sufficient to ensure high-performance operation of R-AWGs. This flatness requirement is not critical and is routinely obtained in standard polishing. When the reflecting surface is either concave or convex, significant additional loss and increased transmission channel spectral width can be seen.

\section{REFERENCES}

[1] A. Himeno, K. Kato, and T. Miya, "Silica-based planar lightwave circuits," IEEE J. Select. Topics Quantum Electron., vol. 4, pp. 913-924, Nov.-Dec. 1998.

[2] M. K. Smit and C. van Dam, "PHASAR-based WDM-devices: Principles, design and applications," IEEE J. Select. Topics Quantum Electron., vol. 2, pp. 236-250, June 1996.

[3] C. Dragone, "Efficient techniques for widening the passband of a wavelength router," J. Lightwave Technol., vol. 16, pp. 1895-1906, Oct. 1998.

[4] M. Zirngibl, C. H. Joyner, and P. C. Chou, "Polarization compensated waveguide grating router on InP," Electron. Lett., vol. 31, pp. 1662-1664, 1995.

[5] C. K. Nadler, E. K. Wildermuth, M. Lanker, W. Hunziker, and H. Melchior, "Polarization insensitive, low-loss, low-crosstalk wavelength multiplexer modules," IEEE J. Select. Topics Quantum Electron., vol. 5, pp. 1407-1412, Sept.-Oct. 1999.

[6] M. B. J. Diemeer, L. H. Spickman, R. Ramsamoedj, and M. K. Smit, "Polymeric phase array wavelength multiplexer operating around 1550 nm," Electron. Lett., vol. 32, pp. 1132-1133, 1996.

[7] Y. Inoue, A. Himeno, K. Moriwaki, and M. Kawachi, "Silica-based arrayed-wave-guide grating circuit as optical splitter router," Electron. Lett., vol. 31, pp. 726-727, 1995.

[8] C. Dragone, "Efficient reflective multiplexer arrangement," U.S. Patent 5450511 , Sept. 12, 1995.

[9] J. B. D. Soole, M. R. Amersfoort, H. P. LeBlanc, A. Rajhel, C. Caneau, C. Youtsey, and I. Adesida, "Compact polarization independent InP reflective arrayed waveguide grating filter," Electron. Lett., vol. 32, pp. 1769-1771, 1996.

[10] L. G. de Peralta, A. A. Bernussi, H. Temkin, M. Borhani, and D. Doucette, "Silicon dioxide waveguides with low birefringence," IEEE J. Quantum Electron., vol. 39, pp. 874-879, July 2003.
[11] L. G. de Peralta, A. A. Bernussi, S. Frisbie, R. Gale, and H. Temkin, "Reflective arrayed waveguide grating multiplexer," IEEE Photon. Technol. Lett., vol. 15, pp. 1398-1400, Oct. 2003.

[12] A. A. Bernussi, L. G. de Peralta, S. Frisbie, and H. Temkin, "Effects of power truncation on the insertion loss and crosstalk of arrayed-waveguide grating devices," Appl. Phys. Lett, vol. 83, pp. 1695-1697, 2003.

[13] K. Okamoto, Fundamentals of Optical Waveguides. New York: Academic, 2000, ch. 9.

[14] C. D. Lee, W. Chen, Q. Wang, Y. J. Chen, W. T. Beard, D. Stone, R. F. Smith, R. Mincher, and I. R. Stewar, "The role of photomask resolution on the performance of arrayed-waveguide grating devices," J. Lightwave Technol., vol. 19, pp. 1726-1733, Nov. 2001.

[15] P. Muñoz, D. Pastor, and J. Capmany, "Modeling and design of arrayed waveguide gratings," J. Lightwave Technol., vol. 20, pp. 661-674, Apr. 2002.

A. A. Bernussi received the B.S., M.S., and Ph.D. degrees in physics from the Universidade Estadual de Campinas, Campinas, Brazil, in 1981, 1984, and 1990, respectively.

In 1988, he joined the Optoelectronic Group at the Brazilian Telecommunication Company (Telebras), where he was involved in the development of semiconductor lasers and materials. From 1994 to 1995, he was a Postdoctoral Researcher at the Electrical Engineering Department, Colorado State University, Fort Collins. His research during this period was on high-temperature properties of strained quantum-well lasers. From 2000 to 2001, he was with the National Synchrotron Light Laboratory (LNLS), Brazil, where he was involved in the development of semiconductor high-power lasers and strained nanostructures. Since 2001, he has been a Research Associate with the Electrical Engineering Department, Texas Tech University, Lubbock, where he is involved in the development, characterization, and packaging of planar lightwave circuits.

L. Grave de Peralta received the M.S. degree in physics from Oriente University, Santiago de Cuba, Cuba, in 1982. and the Ph.D. degree in electrical engineering from Texas Tech University, Lubbock, in 2000.

He was a Professor with the Department of Experimental and Theoretical Physics at Oriente University until 1989. His earlier work focused on verticalcavity surface-emitting lasers (VCSELs) design and material characterization (luminescence and X-ray reflectivity). During the last two years, he has been working in planar lightwave circuit design and development at Texas Tech University.

V. Gorbounov, photograph and biography not available at the time of publication.

J. A. Linn, photograph and biography not available at the time of publication.

S. Frisbie, photograph and biography not available at the time of publication.

R. Gale, photograph and biography not available at the time of publication.

H. Temkin (SM'87-F'93) was a Postdoctoral Associate with Cornell University, Ithaca, NY, where he worked (with Prof. Fitchen) on optical properties of low-dimensional materials from 1975 to 1977. In 1977, he joined the staff of AT\&T Bell Laboratories, Murray Hill, NJ, where he worked on a variety of optoelectronic devices, including light-emitting diodes (LEDs), buried heterostructure lasers, and quantum-well lasers, devices based on SiGe and high-speed bipolar transistors. He became a Distinguished Member of Technical Staff in 1985. In 1992, he joined the faculty of Colorado State University, Fort Collins, where he was Professor of Electrical and Computer Engineering. He moved to Texas Tech University, Lubbock, in 1996, where he serves as a Maddox Chair in Engineering. His research has focused on gas source molecular beam epitaxy (MBE) growth of semiconductors with emphasis on ultraviolet photodetectors and LEDs based on $\mathrm{AlGaN}$ and passive devices for wavelength-division multiplexing. 